\title{
THE SECOND CONTINUOUS JACOBI TRANSFORM
}

\author{
E. Y. DEEBA \\ Department of Applied Mathematical Sciences \\ University of Houston-Downtown \\ Houston, Texas 77002 \\ and \\ E. L. KOH \\ Department of Mathematics and Statistics \\ University of Regina \\ Regina, Canada S4S OA2 \\ (Received February 14, 1984)
}

\begin{abstract}
This paper continues the work started in [1]; a second continuous Jacobi transform is defined for suitable functions $f(x)$. Properties of the transform are studied. In particular, the first continuous Jacobi transform in [1] and the second continuous Jacobi transform are shown to be inverse to each other. The paper concludes with an extension of Campbell's sampling theorem [2].
\end{abstract}

KEY WOROS AND PHRASES: Continuous Jacobi transform, inverse Jacobi transform, discrete Jacobi transform, sampling theorem.

1980 MATHEMATICS SUBJECT CLASSIFICATION CODES. 44A15, 33A30, 94A65.

\section{INTRODUCTION.}

In this paper, the second continuous Jacobi transform, ^ $f^{(\alpha, B)}$, of a function $f(x)$ is developed along similar lines of Butzer, Stens, and Wehrens [3]. The results generalize the work in [3] as well as the work of Debnath [4] on the discrete Jacobi transform. Basic properties of $f(\alpha, \beta)$ will be derived including an inversion formula tersely given by $\left(\mathrm{f}^{(\alpha, \beta)}(\cdot)\right)^{\wedge(\alpha, \beta)}=\mathrm{f}(\cdot)$. The results are then applied to an extended form of Campbel1's sampling theorem [2].

The paper is divided as follows. Section two includes basic notations 
and results obtained in [1] that will be used in the sequel. Section three is devoted to the study of the second continuous Jacobi transform. In this section, the first and the second continuous Jacobi transforms are shown to be inverse to each other. Section four is devoted to a sampling theorem based on Jacobi transforms and an estimate of a truncation error.

2. PRELIMINARIES.

In this section we recall all the necessary background material on Jacobi functions and the first continuous Jacobi transform as studied in [1]. For the sake of completeness, we repeat some of the basic notions of hypergeometric functions.

For each $a, b, c$ real numbers with $c \neq 0,-1,-2, \ldots$ the hypergeometric function is given by

$$
F(a, b ; c ; z)=\sum_{k=0}^{\infty} \frac{(a)_{k}(b) k}{(c)_{k} k !} z^{k},|z|<1
$$

The above series converges absolutely and uniformly on each compact subset of $(-1,1)$. Also,

$$
\lim _{z \rightarrow 1^{-}} F(a, b ; c ; z)=F(a, b ; c ; 1)=\frac{\Gamma(c) \Gamma(c-a-b)}{\Gamma(c-a) \Gamma(c-b)}
$$

where the gamma function is always assumed to be a well-defined function of its argument.

The Jacobi function is defined by

$$
\mathrm{P}_{\lambda}^{(\alpha, \beta)}(\mathbf{x})=\frac{\Gamma(\lambda+\alpha+1)}{\Gamma(\alpha+1) \Gamma(\lambda+1)} \mathrm{F}\left(-\lambda, \lambda+\alpha+\beta+1 ; \alpha+1 ; \frac{1-\mathbf{x}}{2}\right), \quad \mathbf{x} \varepsilon(-1,1]
$$

where $\alpha, \beta>-1$. The following are some of the properties of $p_{\lambda}^{(\alpha, \beta)}(x)$ derived in [1] and will be used in the sequel. We refer the reader to [1] for proofs.

LEMMA 2.1. [Lemma 2.2, [1], page 148]. For any $x \in(-1,1)$ and $\lambda \geq-\frac{\alpha+\beta+1}{2}$, we have for

$$
\begin{aligned}
& \text { (i) }-1<\beta \leq 0,\left|P_{\lambda}^{(\alpha, \beta)}(x)\right| \leq \frac{\Gamma(\lambda+\alpha+1)}{\Gamma(\alpha+1) \Gamma(\lambda+1)}+M(\lambda, \alpha, \beta) \log \left(\frac{2}{1+x}\right) \\
& \text { (ii) } R \geq 0, \quad\left(\frac{1+x}{2}\right){ }^{\beta}\left|P_{\lambda}^{(\alpha, \beta)}(x)\right| \leq \frac{\Gamma(\lambda+\alpha+1)}{\Gamma(\alpha+1) \Gamma(\lambda+1)}+M^{\prime}(\lambda, \alpha, \beta) \log \left(\frac{2}{1+x}\right)
\end{aligned}
$$

where $M(\lambda, \alpha, \beta)$ and $M^{\prime}(\lambda, \alpha, \beta)$ are constants depending on $\lambda, \alpha$, and $\beta$.

We will denote the weighted $L^{p}(-1,1)(p \geq 1)$ space with weight $w(x) \equiv(1-x)^{\alpha}(1+x)^{\beta}$ by $L_{w}^{p}(-1,1)$. The norm on $L_{w}^{p}(-1,1)$ is given by 


$$
\|f\|_{p}=\left(\frac{1}{2^{\alpha+\beta+1}} \int_{-1}^{1} w(x)|f(x)|^{p} d x\right)^{1 / p} .
$$

It was shown in [4] that

LEMMA 2.2. (L emma 2.4, $[1], p .150) \cdot P_{\lambda}^{(\alpha, \beta)}(x) \varepsilon L_{w}^{p}(-1,1)$ for all $p \geq 1$ and for all $\alpha$ and $\beta$ such that $\alpha p+1>0$ and $-1 / p<\beta<1 / p$.

Jacobi polynomials with $\lambda=n \varepsilon P, P$ is the set of all positive integers, satisfy the following orthogonality relation

$$
\frac{1}{2^{\alpha+\beta+1}} \int_{-1}^{1} w(x) P_{n}^{(\alpha, \beta)}(x) P_{m}^{(\alpha, \beta)}(x) d x= \begin{cases}0 & n \neq m \\ \delta_{n} & n=m\end{cases}
$$

where $\delta_{n}=\frac{\Gamma(n+\alpha+1) \Gamma(n+\beta+1)}{n !(2 n+\alpha+\beta+1) \Gamma(n+\alpha+\beta+1)}$.

However, Jacobi functions do not satisfy such an orthogonality relation. Instead, the following result holds:

LEMMA 2.3. (i.emma 2.5, [1], p.150). Let $\lambda$ and $\nu$ both be greater than $-\frac{\alpha+\rho+1}{2}, \lambda \neq v$ and $\lambda \neq-(v+\alpha+\beta+1)$. For $\alpha, \beta \varepsilon\left(-\frac{1}{2}, \frac{1}{2}\right)$, we have

$$
\begin{aligned}
& \frac{1}{2^{\alpha+\rho+1}} \int_{-1}^{1} w(x) P_{\lambda}^{(\alpha, \beta)}(x) P_{v}^{(\beta, \alpha)}(-x) d x \\
& =\frac{\Gamma(\lambda+\alpha+1) \Gamma(\nu+\beta+1)}{\pi(\lambda-v)(\lambda+v+\alpha+\beta+1)}\left\{\frac{\sin \pi \lambda}{\Gamma(v+1) \Gamma(\lambda+\alpha+\beta+1)}-\frac{\text { sin } \pi v}{\Gamma(\lambda+1) \Gamma(v+\alpha+\beta+1)}\right\} .
\end{aligned}
$$

We also recall the definition of the discrete Jacobi transform as studied by Debnath in [4].

$$
\hat{f}^{(\alpha, E)}(n)=J(f(x))=\frac{1}{2^{\alpha+\beta+1}} \int_{-1}^{1} w(x) P_{n}^{(\alpha, \beta)}(x) f(x) d x
$$

and under appropriate conditions

$$
f(x)=\sum_{n=0}^{\infty} \delta_{n}^{-1 \hat{f}}(\alpha, \beta)(n) P_{n}^{(\alpha, \beta)}(x)
$$

Now, for any $f, g \in L_{w}^{2}(-1,1)$ and for appropriate $\alpha, \beta$

$$
\frac{1}{2^{\alpha+\rho+1}} \int_{-1}^{1} w(x) f(x) g(x) d x=\sum_{n=0}^{\infty} \delta_{n}^{-1 \hat{f}}(\alpha, \beta)(n) \hat{g}^{(\alpha, \beta)}(n) .
$$

From (2.3) and Lemma 2.3 together with the identity $P_{n}^{(\alpha, \beta)}(-x)=(-1){ }_{P_{n}}^{(\beta, \alpha)}(x)$, we obtain

$$
\hat{P_{\lambda}^{(\alpha, \beta)}}(n)= \begin{cases}(-1)^{n} \frac{\Gamma(\lambda+\alpha+1) \Gamma(n+\beta+1) \sin \pi \lambda}{\pi(\lambda-n)(\lambda+n+\alpha+\beta+1) n ! \Gamma(\lambda+\alpha+\beta+1)}, & \lambda \neq n \\ \frac{\Gamma(n+\alpha+1) \Gamma(n+\beta+1)}{(2 n+\alpha+\beta+1) n ! \Gamma(n+\alpha+\beta+1)} & , \lambda=n\end{cases}
$$


The following estimates on $P_{\lambda}^{(\alpha, \beta)}(x)$ for large $\lambda$ will be used.

LEMMA 2.4. (Lemma 2.6, [1], page 152). For $\lambda, v \geq-\frac{\alpha+\beta+1}{2}, \alpha>-\frac{1}{2}$ and $-\frac{1}{2}<R<\frac{1}{2}$, we have

(i) for each $[a, b] \subset(-1,1)$, there holds for all $x \varepsilon[a, b]$

$$
\left|P_{\lambda}^{(\alpha, \beta)}(\mathbf{x})\right|=O\left(\lambda^{-\frac{1}{2}}\right) \text { as } \lambda \rightarrow \infty \text {. }
$$

(ii) $\left\|P_{\lambda}^{(\alpha, \beta)}(x)\right\|_{1} \leq\left\|P_{\lambda}^{(\alpha, \beta)}(x)\right\|_{2}=O\left(\lambda^{-\frac{1}{2}}\right)$ as $\lambda \rightarrow \infty$.

(iii) for each $[c, d] \subseteq\left[-\frac{\alpha+\beta+1}{2}, \infty\right)$, there exists a constant $M>0$ such that for all $\lambda, v \varepsilon[c, d]$

$$
\left\|\mathbf{P}_{\lambda}^{(\alpha, R)}(\mathbf{x})-\mathbf{P}_{\nu}^{(\alpha, \beta)}(\mathbf{x})\right\|_{2} \leq \mathbf{M}|\lambda-\nu|
$$

The continuous Jacobi transform of the first kind is defined as in

[1] by

$$
\hat{f}_{(\lambda)}^{(\alpha, \beta)}=\frac{1}{2^{\alpha+\beta+1}} \int_{-1}^{1} w(x) P_{\lambda}^{(\alpha, \beta)}(x) f(x) d x .
$$

for every $f \varepsilon L_{w}^{2}(-1,1)$ with $\alpha>-\frac{1}{2}$ and $-\frac{1}{2}<\beta<\frac{1}{2}$. We recall that if $\alpha=\beta=0$, we obtain the results of Butzer, Stens and Wehrens [3] and if $\lambda=n \varepsilon P$, we obtain the results of Debnath [4].

Azain, it was shown in $[1]$ that for any $f \in L_{w}^{2}(-1,1)$ we have

$$
\left|\hat{f}^{(\alpha, \beta)}(\lambda)\right|=0\left(\lambda^{-\frac{1}{2}}\right) \quad \text { as } \lambda \rightarrow \infty \text {. }
$$

and

$$
\hat{f}^{(\alpha, \beta)}\left(\cdot-\frac{\alpha+\beta+1}{2}\right) \varepsilon C_{0}\left(\mathbb{R}^{+}\right) \cap L^{p}\left(\mathbb{R}^{+}\right), p>2 .
$$

The following Lemma will be essential for our work.

LEMMA 2.5. (Lemma 3.2, [1], page 155). Let $F(x)$ be defined on $\mathbb{R}^{+}=[0, \infty)$ such that $\lambda^{\alpha+\beta+\frac{1}{2}} \quad r(\lambda) \in L^{1}\left(\mathbb{R}^{+}\right)$. Then the function

$$
G(x)=\int_{0}^{\infty} F(\lambda) P{ }_{\lambda-\frac{\alpha+\beta+1}{2}}^{(\beta, \alpha)}(-x) H(\lambda) \lambda \sin \pi \lambda d \lambda
$$

belongs to $C(-1,1) \cap \mathrm{L}_{\mathrm{w}}^{2}(-1,1)$ where

$$
H(\lambda)=\frac{\Gamma^{2}\left(\lambda+\frac{\alpha+\beta+1}{2}\right)}{\Gamma\left(\lambda+\frac{\alpha-\beta+1}{2}\right) \Gamma\left(\lambda+\frac{\beta-\alpha+1}{2}\right)} .
$$

From now on we will assume throughout the paper that $\alpha$ and $\beta$ satisfy 


$$
\alpha+\beta=0,-\frac{1}{2}<\alpha, \quad \beta<\frac{1}{2} \text {. }
$$

If $\alpha$ and $\beta$ satisfy (2.8), then it was shown in [1] that

$$
\mathbf{P}_{k}^{(\alpha, \beta)}(x)=4 \int_{0}^{\infty} \hat{F}_{k}^{(\alpha, \beta)}\left(\lambda-\frac{1}{\alpha}\right) \mathrm{P}_{\lambda-\frac{1}{2}}^{(\beta, \alpha)}(-x) \mathrm{H}_{0}(\lambda) \lambda \sin \pi \lambda d \lambda
$$

where

$$
H_{0}(\lambda)=\frac{\Gamma^{2}\left(\lambda+\frac{1}{2}\right)}{\Gamma\left(\lambda+\alpha+\frac{1}{2}\right) \Gamma\left(\lambda+\beta+\frac{1}{2}\right)}, x \in(-1,1], k \in P .
$$

The following is also true.

LEIMA 2.6. (Theorem 3.1, [1], page 158). Let $f \in L_{w}^{2}(-1,1)$ be such that $\lambda^{\frac{1}{2} \hat{f}} \hat{f}(\alpha, \beta)\left(\lambda-\frac{1}{2}\right) \in L^{1}\left(\mathbb{R}^{+}\right)$. Then for almost every $x \in(-1,1)$

$$
f(x)=4 \int_{0}^{\infty} \hat{f}(\alpha, \beta)\left(\lambda-\frac{1}{2}\right) P_{\lambda-\frac{1}{2}}^{(\beta, \alpha)}(-x) H_{0}(\lambda) \lambda \sin \pi \lambda d \lambda .
$$

Moreover, if $f(x)$ is continuous then (2. D) holds everywhere on $(-1,1)$.

3. SECOND CONTINUOUS JACOBI TRANSFORM

In this section we define the second continuous Jacobi transform ${ }^{a} f(\alpha, \beta)$ of a function $f$ with $\alpha$ and $\beta$ satisfying (2.8). We also show that $\wedge_{f}(\alpha, \beta)$ and $f^{\wedge}(\alpha, \beta)$ are inverse of each other.

For each $f$ defined on $\mathrm{IR}^{+}$, we associate the integral

$$
I(x)=4 \int_{0}^{\infty} f(\lambda) P_{\lambda-\frac{1}{2}}^{(\beta, \alpha)}(-x) \frac{\Gamma\left(\lambda+\frac{1}{2}\right)}{\Gamma\left(\lambda+\beta+\frac{1}{2}\right)} \lambda \sin \pi \lambda d \lambda .
$$

Proposition 3.1. For each $f$ defined on $R^{+}$with $f$ and $\lambda^{-\beta+\frac{1}{2}} f \in L^{1}\left(I R^{+}\right)$, we have $I(x) \varepsilon C(-1,1) \cap L_{w}^{2}(-1,1)$. 'Although the proof of Proposition 3.1 goes along the same lines as Lema 2.5, we present the proof with the proper modification for the sake of self-containment.

PROOF of Proposition 3.1. We first show that $I(x) 18$ well defined. Observe that, for large $\lambda, \frac{\Gamma\left(\lambda+\frac{1}{2}\right)}{\Gamma\left(\lambda+\beta+\frac{1}{2}\right)}$ behaves like $\lambda^{-\beta}$ (see [1]). Thus for any fixed $T>0$, we have by Lemma 2.4 (1i) and the hypothesis that

$$
\begin{aligned}
|I(x)| \leq\left|4 \int_{0}^{T} f(\lambda) P_{\lambda-\frac{1}{2}}^{(\beta, \alpha)}(-x) \frac{\Gamma\left(\lambda+\frac{1}{2}\right)}{\Gamma\left(\lambda+\beta+\frac{1}{2}\right)} \lambda \sin \pi \lambda d \lambda\right| \\
+\left|4 \int_{T}^{\infty} f(\lambda) P_{\lambda-\frac{1}{2}}^{(\beta, \alpha)}(-x) \frac{\Gamma\left(\lambda+\frac{1}{2}\right)}{\Gamma\left(\lambda+\beta+\frac{1}{2}\right)} \lambda \sin \pi \lambda d \lambda\right| \\
\quad \leq C_{1}+C_{2} \int_{T}^{\infty} \lambda^{-\beta+\frac{1}{2}}|f(\lambda)| d \lambda<\infty
\end{aligned}
$$


where $C_{1}$ and $C_{2}$ are some positive constants. Hence $I$ is well-defined. We show that $I(x) \in C(-1,1)$. For any $x \in(-1,1)$, there exists a $\delta_{1}>0$ such that $x \pm \delta_{1} \varepsilon(-1,1)$. By lemma 2.4 (1) and for all $|y|<\delta_{1}$, we have

$$
\begin{aligned}
|I(x+y)-I(x)| & \leq\left|4 \int_{0}^{\infty} f(\lambda)\left(P_{\lambda-\frac{1}{2}}^{(\beta, \alpha)}(-x-y)-P_{\lambda-\frac{1}{2}}^{(\beta, \alpha)}(-x)\right) \frac{\Gamma\left(\lambda+\frac{1}{2}\right)}{\Gamma\left(\lambda+\beta+\frac{1}{2}\right)} \lambda \sin \pi \lambda d \lambda\right| \\
& \leq 8 \mathrm{C} \int_{0}^{\infty} \lambda^{-\beta+\frac{1}{2}}|f(\lambda)| d \lambda<\infty
\end{aligned}
$$

by hypothesis and $C$ is some positive constant. Thus for $\lambda_{0}$ sufficiently large.

$$
\mid 4 \int_{\lambda_{0}}^{n} f(\lambda)\left(P_{\lambda-\frac{1}{2}}^{(\beta, \alpha)}(-x-y)-P_{\lambda-\frac{1}{2}}^{(\beta, \alpha)}(-x) \frac{\Gamma\left(\lambda+\frac{1}{2}\right)}{\Gamma\left(\lambda+\beta+\frac{1}{2}\right)} \lambda \sin \pi \lambda d \lambda \mid\right.
$$

can be made sufficiently small. Thus for $\varepsilon>0$ given, there exists a $\lambda_{0}$ sufficiently large such that the above difference is less than $\varepsilon / 2$. Fix $\lambda_{0}$. By the continuity of $P_{\lambda-\frac{1}{2}}^{(\beta, \alpha)}(-x)$, we have for $\hat{\varepsilon}>0$ that there exists a $\delta_{2}>0$ such that

$$
\left|P_{\lambda \rightarrow \frac{1}{2}}^{(\beta, \alpha)}(-x-y)-P_{\lambda-\frac{1}{2}}^{(\beta, \alpha)}(-x)\right|<\hat{\varepsilon} \text { whenever }|y|<\delta_{2} .
$$

Choose $\varepsilon=2 \hat{\varepsilon} \int_{0}^{\lambda} 0_{\lambda}-\beta+\frac{1}{2}|f(\lambda)| d \lambda$ and $\delta=\min \left(\delta_{1}, \delta_{2}\right)$. With this choice. $|I(x+y)-I(x)|<\varepsilon$, whenever $|y|<\delta$. Therefore $I(x) \varepsilon C(-1,1)$.

We finally show that $I(x) \in L_{w}^{2}(-1,1)$.

$$
\|I(x)\|_{2}^{2}=\frac{1}{2} \int_{-1}^{1} w(x)|I(x)|^{2} d x
$$

and by Hardy-Littlewood inequality (see [1], page 148) and Lemma 2.4 (11), we have

$$
\begin{aligned}
\|I(x)\|_{2} & \leq \int_{0}^{\infty} \lambda^{-\beta+\frac{1}{2}}|f(\lambda)| d \lambda\left(\left.\frac{1}{2}\right|_{-1} ^{1}(1-x)^{\alpha}(1+x)^{\beta}\left|P_{\lambda-\frac{1}{2}}^{(\beta, \alpha)}(-x)\right|^{2} d x\right)^{\frac{1}{2}} \\
& \leq \int_{0}^{\infty} \lambda^{-\beta+\frac{1}{2}}|f(\lambda)| d \lambda<\infty
\end{aligned}
$$

by hypothesis. Hence $I(x) \varepsilon C(-1,1) \cap L_{w}^{2}(-1,1)$. This completes the proof of Proposition 3.1.

We shall call $I(x)$ the second continuous Jacobi transform and we will denote this by $\wedge_{f}^{(\alpha, \beta)}$. Thus, we define

$$
\lambda_{f}(\alpha, \beta)(x)=4 \int_{0}^{\infty} f(\lambda) P_{\lambda-\frac{1}{2}}^{(\beta, \alpha)}(-x) \frac{\Gamma\left(\lambda+\frac{1}{2}\right)}{\Gamma\left(\lambda+\beta+\frac{1}{2}\right)} \quad \lambda \sin \pi \lambda d \lambda .
$$


From Proposition 3.1 we deduce that ${ }^{\wedge} f(\alpha, \beta) \in C(-1,1) \cap L_{w}^{2}(-1,1)$ with $-\frac{1}{2}<\alpha, \beta<\frac{1}{3}$ and $\alpha+f=0$. In the next theorem, we will prove an inversion formula for the transform $\wedge_{f}(\alpha, \beta)$ under appropriate choice of $f$. The inversion formula proof is analogous to the proof given in [3]. We will employ the Fourier cosine transform of a function $f$ which is given by

$$
F_{C}(f)(t)=\sqrt{\frac{2}{\pi}} \int_{0}^{\infty} f(\lambda) \cos \lambda t(1)
$$

THEOREM 3.1. As sume that $f \in L^{1}\left(I^{+}\right)$is such that $\lambda^{-B+\frac{1}{s}} f(\lambda) \varepsilon L^{1}\left(\mathbb{R}^{+}\right)$ and that

$$
F_{C}(f)(t) \equiv 0 \text { for all } \pi \leq t<\infty \text {. }
$$

Then we have for almost all $\lambda \in \mathbb{R}^{+}$

$$
\frac{1}{2} \frac{\Gamma\left(\lambda+\frac{1}{2}\right)}{\Gamma\left(\lambda+\alpha+\frac{1}{2}\right)} \int_{-1}^{1} w(x)^{\wedge} f(\alpha, \beta)(x) P_{\lambda-\frac{1}{2}}^{(\alpha, \beta)}(x) \mathrm{d} x=f(\lambda) .
$$

Moreover, if $f \in C\left(\mathbb{R}^{+}\right)$, then (3.1) holds for all $\lambda \in \mathbb{R}^{+}$.

PROOF. By employing Fubini's theorem together with Lemma 2.3

(with $\alpha+\ell=0$ ), we obtain

$$
\begin{aligned}
& \frac{1}{2} \frac{\Gamma\left(\lambda+\frac{1}{2}\right)}{\Gamma\left(\lambda+\alpha+\frac{1}{2}\right)} \int_{-1}^{1} w(x)\left\{4 \int_{0}^{\infty} f(t) P_{t-\frac{1}{2}}^{(\beta, \alpha)}(-x) \frac{\Gamma\left(t+\frac{1}{2}\right)}{\Gamma\left(t+\beta+\frac{1}{2}\right)} t \sin \pi t d t\right\} P_{\lambda-\frac{1}{2}}^{(\alpha, \beta)}(x) d x \\
& =\frac{2 \Gamma\left(\lambda+\frac{1}{2}\right)}{\Gamma\left(\lambda+\alpha+\frac{1}{2}\right)} \int_{0}^{\infty} t f(t) \sin \pi t \frac{\Gamma\left(t+\frac{1}{2}\right)}{\Gamma\left(t+\beta+\frac{1}{2}\right)}\left\{\int_{-1}^{1} w(x) P_{\lambda-\frac{1}{2}}^{(\alpha, \beta)}(x) P_{t-\frac{1}{2}}^{(\beta, \alpha)}(-x) d x\right\} d t \\
& =4 \frac{\Gamma\left(\lambda+\frac{1}{2}\right)}{\Gamma\left(\lambda+\alpha+\frac{1}{2}\right)} \int_{0}^{\infty} t f(t) \sin \pi t \frac{\Gamma\left(t+\frac{1}{2}\right)}{\Gamma\left(t+\beta+\frac{1}{2}\right)} \frac{\Gamma\left(\lambda+\alpha+\frac{1}{2}\right) \Gamma\left(t+\beta+\frac{1}{2}\right)}{\pi\left(\lambda^{2}-t^{2}\right) \Gamma\left(t+\frac{1}{2}\right) \Gamma\left(\lambda+\frac{1}{2}\right)} \\
& =4 \int_{0}^{\infty} t f(t) \sin \pi t\left\{\frac{\cos \pi t-\cos \pi \lambda}{\pi\left(\lambda^{2}-t^{2}\right)} d t \equiv Q(\lambda) .\right.
\end{aligned}
$$

Set $q_{t}(\lambda)=\frac{\cos \pi t-\cos \pi \lambda}{\pi\left(\lambda^{2}-t^{2}\right)}$.

We claim that $Q(\lambda) \in L^{1}\left(\mathbb{R}^{+}\right)$. Indeed, an application of Fubini's theorem together with the assumption that $f \in L^{1}\left(\mathbb{R}^{+}\right)$implies that

$$
\int_{0}^{\infty}|Q(\lambda)| d \lambda<\infty
$$

Now, by another application of Fubini's theorem, we obtain 


$$
\begin{aligned}
& F_{C}[Q](s)=\sqrt{\frac{2}{\pi}} \int_{0}^{\infty} Q(\lambda) \cos \lambda s d \lambda \\
& =\sqrt{\frac{2}{\pi}} \int_{0}^{\infty}\left(4 \int_{0}^{\infty} t f(t) \sin \pi t q_{t}(\lambda) d t\right) \cos \lambda s d \lambda \\
& =4 \sqrt{\frac{2}{\pi}} \int_{0}^{\infty} t f(t) \sin \pi t\left(\int_{0}^{\infty} q_{t}(\lambda) \cos \lambda s d \lambda\right) d t \\
& =4 \int_{0}^{\infty} t f(t) \sin \pi t F_{C}\left(q_{t}\right)(s) d t .
\end{aligned}
$$

We employ the result of [3] and proceed analogously and determine that

$$
F_{C}\left(q_{t}\right)(s)=\sqrt{\frac{2}{\pi}} \frac{1}{2 t} \sin (\pi-s) t, \quad 0 \leq s \leq \pi, t>0
$$

Thus

$$
\begin{aligned}
& F_{C}(Q)(s)=2 \sqrt{\frac{2}{\pi}} \int_{0}^{\infty} f(t) \sin \pi t \sin (\pi-s) t d t \\
& F_{C}(Q)(s)=F_{C}(f)(s)-F_{C}(f)(2 \pi-s)=F_{C}(f)(s), 0 \leq s \leq \pi .
\end{aligned}
$$

Moreover, $F_{C}(Q)(s)=0$ for $\pi \leq s<\infty$. The assumption $F_{C}(f)(s)=0$ for $\pi \leq s<\infty$ together with the uniqueness of the Fourier-cosine transform implies that $Q(\lambda)=f(\lambda)$ for almost every $\lambda \varepsilon \mathbb{R}^{+}$. Thus

$$
f(\lambda)=\frac{1}{2} \frac{\Gamma\left(\lambda+\alpha+\frac{1}{2}\right)}{\Gamma\left(\lambda+\frac{1}{2}\right)} \int_{-1}^{1} w(x)^{\wedge} f^{(\alpha, \beta)}(x) P_{\lambda-\frac{1}{2}}^{(\alpha, \beta)}(x) d x .
$$

The continuity of $f$ will imply that (3.1) holds for all $\lambda \in \mathbb{R}^{+}$.

From Theorem 3.1 and Lemma 2.6 we deduce that

$$
\left(\wedge f^{(\alpha, \beta)}(\cdot)\right)^{\wedge}(x, \beta)(\lambda)=2 \frac{\Gamma\left(\lambda+\alpha+\frac{1}{2}\right)}{\Gamma\left(\lambda+\frac{1}{2}\right)} f(\lambda) .
$$

and

$$
\wedge\left(\frac{\Gamma\left(\lambda+\frac{1}{2}\right)}{\Gamma\left(\lambda+\alpha+\frac{1}{2}\right)} f^{\wedge}(\alpha, \beta)(\cdot)\right){ }^{(\alpha, \beta)}(x)=f(x)
$$

Equations (3.2) and (3.3) reduce to the formulas obtained in [3] whenever $\alpha=\beta=0$.

\section{A SAMPLING THEOREM}

In this section we give a proof of a sampling Theorem of Campbel1 [2] by employing results on the continuous Jacobl transform of the first and second kinds. Moreover, we will obtain an error estimate for a function $f$ that is band-limited in the sense of the Fourier-cosine transform. 
THEOREM 4.1. If $\mathrm{FEC}\left(\mathrm{IR}^{+}\right)$is given by

$$
F(\lambda)=\frac{1}{2} \int_{-1}^{1} w(x) f(x) P_{\mu \lambda-\frac{1}{2}}^{(\alpha, \beta)}(x) d x
$$

for some $\mu>0$ and $f \varepsilon L_{w}^{2}(-1,1)$, then one obtains for all $\lambda \varepsilon \mathbb{R}^{+}$that

$$
F(\lambda)=\sum_{n=0}^{\infty} \frac{(2 n+1) \Gamma(n+1) \Gamma\left(\mu \lambda+\alpha+\frac{1}{2}\right) F\left(\frac{n+\frac{1}{2}}{\mu}\right)}{\pi\left(\lambda^{2} \mu^{2}-\left(n+\frac{1}{2}\right)^{2}\right) \Gamma(n+x+1) \Gamma\left(\lambda \mu+\frac{1}{2}\right)} \sin \pi\left(\lambda \mu-\left(n+\frac{1}{2}\right)\right)
$$

PROOF. From (4.1), (2.3) and (2.4), we obtain (with $\alpha+\beta=0$ )

$$
\begin{aligned}
F(\lambda) & =\sum_{n=0}^{\infty} \frac{n !(2 n+1) \Gamma(n+1)}{\Gamma(n+\alpha+1) \Gamma(n+\beta+1)} \hat{f}^{(\alpha, \beta)}(n) \hat{P}_{\mu \lambda-\frac{1}{2}}^{(\alpha, \beta)}(n) \\
& =\sum_{n=0}^{\infty} \frac{(-1)^{n} n !(2 n+1) \Gamma(n+1)}{\Gamma(n+\alpha+1) \Gamma(n+\beta+1)} \frac{\Gamma\left(\lambda \mu+\alpha+\frac{1}{2}\right) \Gamma(n+\beta+1) \sin \pi\left(\lambda \mu-\frac{1}{2}\right)}{\pi\left(\lambda^{2} \mu^{2}-\left(n+\frac{1}{2}\right)^{2}\right) n ! \Gamma\left(\lambda \mu+\frac{1}{2}\right)} \hat{f}^{(\alpha, \beta)}(n) \\
& =\sum_{n=0}^{\infty} \frac{(-1)^{n}(2 n+1) \Gamma(n+1) \Gamma\left(\lambda \mu+\alpha+\frac{1}{2}\right)}{\pi\left(\lambda^{2} \mu^{2}-\left(n+\frac{1}{2}\right)^{2}\right) \Gamma(n+\alpha+1) \Gamma\left(\lambda \mu+\frac{1}{2}\right)} \sin \pi\left(\lambda \mu-\frac{1}{2}\right) F\left(\frac{n+1}{2}\right)
\end{aligned}
$$

or

$$
F(\lambda)=\sum_{n=0}^{\infty} \frac{(2 n+1) \Gamma(n+1) \Gamma\left(\lambda \mu+\alpha+\frac{1}{2}\right) \sin \pi\left(\lambda \mu-\left(n+\frac{1}{2}\right)\right)}{\pi\left(\lambda^{2} \mu^{2}-\left(n+\frac{1}{2}\right){ }^{2}\right) \Gamma(n+\alpha+1) \Gamma\left(\lambda \mu+\frac{1}{2}\right)} F\left(\frac{n+\frac{1}{2}}{\mu}\right) .
$$

REMARK 4.1. Theorem 3.1 when applied to the above situation yields the series representation $(4.2)$ for $F \in L^{1}\left(\mathbb{R}^{+}\right), \lambda^{-\beta+\frac{1}{2}} F \varepsilon L^{1}\left(\mathbb{R}^{+}\right)$and $F_{C}(F)(s)=0$ for $s>\pi \mu$ for some $\mu>0$.

Let $S_{n, \mu} F$ denote the $n$-th partial sum of the series in (4.2). We give below an error estimate for approximating F. In particular, we show that

THEOREM 4.2. Let F satisfy hypotheses of Theorem 4.1 for some fixed $\mu>0$. Then there exists a constant $c>0$ such that

$$
\left|F(\lambda)-\left(S_{n, \mu} F\right)(\lambda)\right| \leq C(\lambda+1)^{\frac{1}{2}} E_{n}(f)
$$

where

$$
E_{n}(f)=\underset{P_{n} \in P}{=i n f}\left\|f-P{ }_{n}\right\|_{2}
$$

$P_{n}$ is the set of all algebraic polynomials of degree $\pi$.

PROOF. Denote by $s_{n} f$ the $n-t h$ partial sum of

$$
f(x) \simeq \sum_{n=0}^{\infty} \delta_{n}^{-1} \hat{f}^{(\alpha, \beta)}(n) P_{n}^{(\alpha, \beta)}(x)
$$


where $\delta_{n}^{-1}=\frac{(n !)^{2}(2 n+1)}{\Gamma(n+\alpha+1) \Gamma(n+\ell+1)}$.

Thus

$$
\left(S_{n, \mu} F\right)(\lambda)=\sum_{k=0}^{\infty} \frac{(2 k+1) \Gamma(k+1) \Gamma\left(\lambda \mu+\alpha+\frac{1}{2}\right)}{\pi\left(\lambda^{2} \mu^{2}-\left(k+\frac{1}{2}\right)^{2}\right) \Gamma(k+\alpha+1) \Gamma\left(\lambda \mu+\frac{1}{2}\right)}\left(S_{n} f\right)^{\wedge}(\alpha, \beta) \hat{p}_{\lambda \mu-\frac{1}{2}}^{(\alpha, \beta)}(k) .
$$

By Cauchy-Schwartz inequality and Lemma 2.4, we have

$$
\begin{aligned}
\mid F(\lambda) & -\left.\left(S_{n, \mu} F\right)(\lambda)\right|^{2}=\left|\frac{1}{2} \int_{-1}^{1} w(x)\left(f(x)-\left(S_{n} f\right)(x)\right) P_{\lambda \mu-\frac{1}{2}}^{(\alpha, \beta)}(x) d x\right|^{2} \\
& \leq \frac{1}{2} \int_{-1}^{1} w(x)\left|f(x)-\left(S_{n} f\right)(x)\right|^{2} d x \cdot \frac{1}{2} \int_{-1}^{1} w(x)\left|P_{\lambda \mu-\frac{1}{2}}^{(\alpha, \beta)}(x)\right|^{2} d x \\
& \leq\left\|f-S_{n} f\right\|_{2}^{2} \mid P_{\lambda \mu-\frac{1}{2}}^{(\alpha, \beta)}\left\|_{2}^{2} \leq E_{n}^{2}(f)\right\| P_{\mu \lambda-\frac{1}{2}} \|_{2}^{2} .
\end{aligned}
$$

Thus,

$$
\left|F(\lambda)-\left(S_{n, \mu} F\right)(\lambda)\right| \leq C E_{n}(f)(\lambda+1)^{-\frac{1}{2}} \text { for some constant } C \text {. }
$$

REMARK 4.2 The estimate obtained in Theorem 4.2 shows that the error becomes smaller for large $\lambda$.

ACKNOWLEDGEMENT. The work of the first author was partially supported by a grant from the University of Houston-Downtown while the work of the second author was partially supported by NSERC of Canada under Grant A-7184.

\section{References}

1. DEEBA, E.Y. and KOH, E.L. The Continuous Jacobi Transform, Internat. J. Math. \& Math. Sci., Vol. 6, No. 1 (1983), 145-160.

2. CAMPBELL, L.L. A Comparison of the Sampling Theorems of Kramer and Whittaker, J. Soc. Induct. Appl. Math. 12 (1964), 117-130.

3. BUTZER, P.L., STENS, R.L. and WEHRENS, M. Continuous Legendre Transform, Internat. J. Math. \& Math. Sci., Vol. 3, No. 1 (1980), 47-67.

4. DEBNATH, L. On Jacob1 Transform, Bull. Cal. Math. Soc., Vol. 55 (1963) 113-120.

5. ERDELYI, A. MAGNUS, W., OBERHETTINGER F. and TRICOMI, F.G. Higher Transcendental Functions, Vol. 1, McGraw H111, New York $\overline{1953}$. 


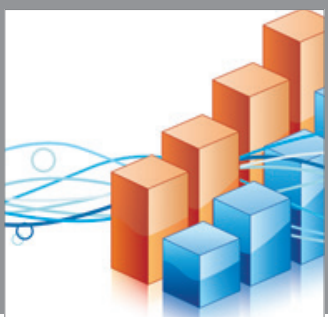

Advances in

Operations Research

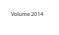

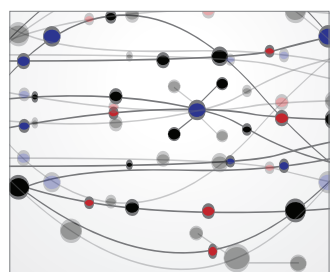

\section{The Scientific} World Journal
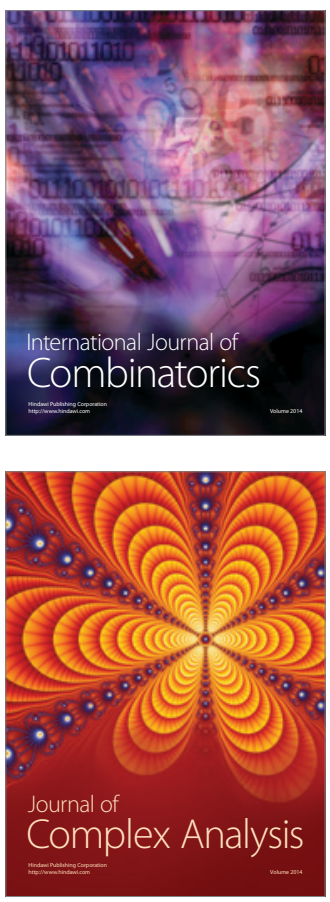

International Journal of

Mathematics and

Mathematical

Sciences
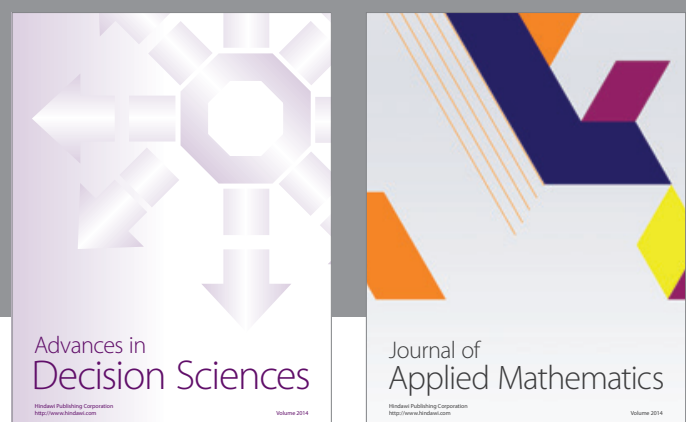

Journal of

Applied Mathematics
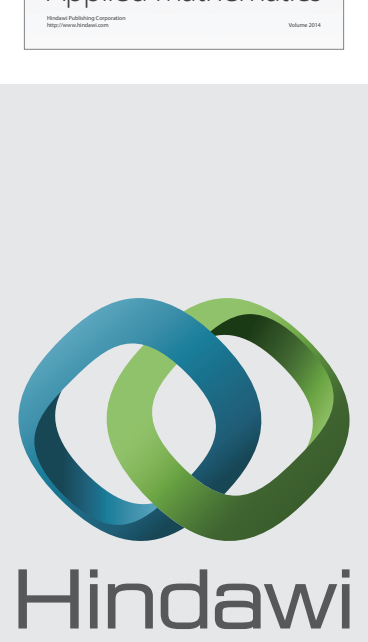

Submit your manuscripts at http://www.hindawi.com
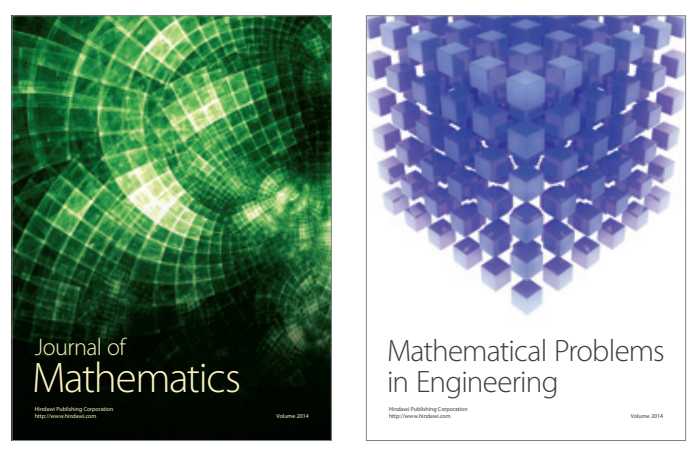

Mathematical Problems in Engineering
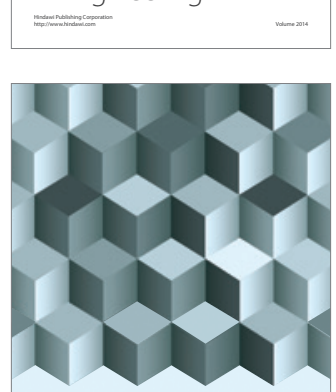

Journal of

Function Spaces
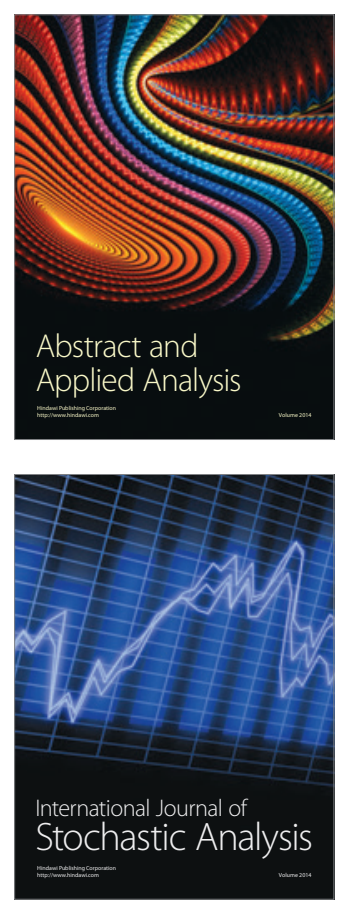

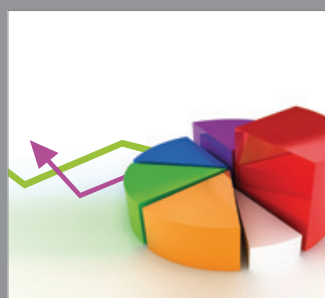

ournal of

Probability and Statistics

Promensencen
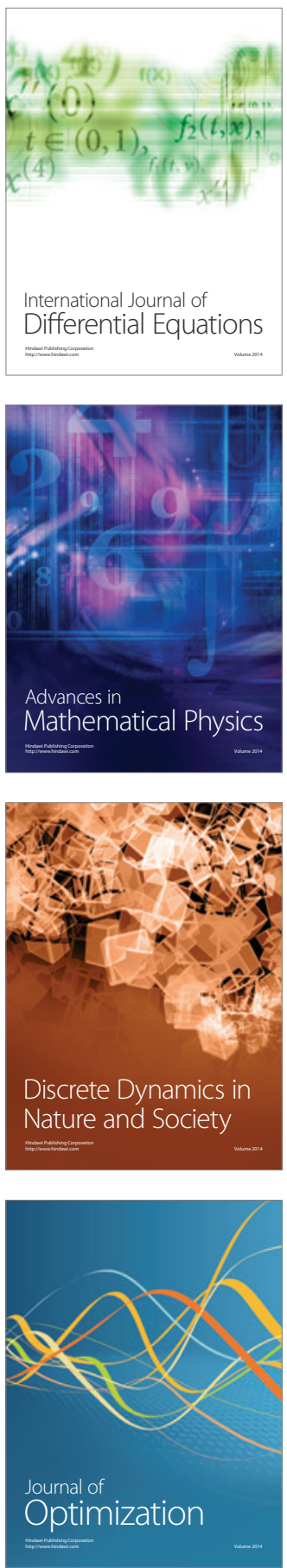